\section{Transpupillary thermotherapy for the treatment of choroidal neovascularization in age-related macular degeneration in Taiwan}

T-H Tsai, C-M Yang, C-H Yang, T-C Ho, J-S Huang and M-S Chen recurrence was found independent of $\mathrm{CNV}$ type. Most improved vision was found mostly in classic CNV. Complications associated with high energy level should be considered in light-brown retinas.

Eye (2007) 21, 721-726; doi:10.1038/sj.eye.6702312; published online 17 March 2006

Keywords: transpupillary thermotherapy; agerelated macular degeneration; choroidal neovascularization

\section{Introduction}

Age-related macular degeneration (AMD) is one of the leading causes of blindness in patients older than 65 years of age. An estimated $80 \%$ of severe vision loss occurs secondary to the formation of choroidal neovascularization $(\mathrm{CNV}){ }^{1,2}$ Laser photocoagulation has been shown to be effective in the treatment of well-defined choroidal neovascular membranes outside of the fovea in the macular photocoagulation study (MPS). However, many lesions are subfoveal or are ill-defined. ${ }^{3,4}$ One study showed that only $13 \%$ of patients with CNV resulted from AMD are eligible for treatment by MPS criteria. ${ }^{5}$ Recently, photodynamic therapy (PDT) has shown benefit in treating $\mathrm{CNV}$ in AMD, primarily of predominantly classic type lesions. Occult lesions responded less favourably. ${ }^{6}$ Moreover, multiple sessions are usually necessary regardless of CNV types, ${ }^{7}$ and the cost of photosensitizing agent is high.
Department of Ophthalmology, National Taiwan University Hospital, Taipei, Taiwan

Correspondence: C-M Yang, Department of Ophthalmology, National Taiwan University Hospital, No. 7, Chung-Shan South Road, Taipei 100, Taiwan Tel: + 886223123456 ext. 5187

Fax: +886223412875

E-mail: chungmay@

ha.mc.ntu.edu.tw

Received: 1 December 2005 Accepted in revised form: 29 January 2006 Published online: 17 March 2006

Proprietary interest: none 
Utilization of transpupillary thermotherapy (TTT) in the treatment of occult CNV secondary to AMD was first reported in $1999 .{ }^{8}$ Unlike conventional short-pulse photocoagulation, which generates local temperature increases of $40-60^{\circ} \mathrm{C}$, TTT gives temperature rises of approximately $10^{\circ} \mathrm{C}$ as predicted by thermal modelling. It also causes less overlying tissue damage for typical clinical parameters used in CNV treatment. ${ }^{9}$ Several studies show encouraging results of TTT in the stabilization or improvement of visual acuity, as well as its effectiveness in CNV closure. ${ }^{8,10,11}$ However, most of the study participants were Caucasian, and the recurrence rate of CNV after TTT has not been well studied.

The aim of the study was to evaluate the therapeutic outcome and the recurrence rate of CNV secondary to AMD after TTT treatment in light-brown retinas.

\section{Patients and methods}

All study patients were treated between August 2000 and May 2004. Patients were 55 years of age or older, and diagnosed with exudative type AMD with subfoveal CNV. Patients with visual acuity less than 20/50 were included in the study. Patients who had received previous photocoagulation or were on alternative treatment for AMD were excluded from the study. In addition, patients with other ocular diseases associated with CNV, such as high myopia, angioid streaks, idiopathic polypoidal choroidovasculopathy, intraocular inflammation, or trauma, were also excluded.

All patients provided informed consent to receive TTT. At the initial visit and all subsequent follow-up visits, the patients had a general ophthalmologic examination including Snellen visual acuity, anterior segment examination, and biomicroscopic examination of the macula with a 78 or $90 \mathrm{D}$ Volk lens (Volk Optical Inc., Mentor, $\mathrm{OH}, \mathrm{USA}$ ) under mydriasis with $1 \%$ tropicamide. Fluorescein angiography (Topcon, Tokyo, Japan) (sodium fluorescein $5 \mathrm{ml}, 10 \%$ ) and colour fundus photography (Topcon, Tokyo, Japan) were performed in all patients at initial visit and at follow-up visits. Indocyanine green angiography (Topcon, Tokyo, Japan) was performed in selected patients before and after treatment. A neovascularization membrane with welldelineated border was defined as a classic membrane; fibrovascular retinal pigment epithelium (RPE) detachment or any membrane without distinct margin was defined as occult. Membranes that contained both components regardless of individual composition were defined as mixed.

TTT was delivered via a slit-lamp using an infrared diode laser at $810 \mathrm{~nm}$ (Iris Medical OcuLight SLx, Iridex Corporation, Mountain View, CA, USA). Topical 0.5\% proparacaine hydrochloride was applied to the eye before placement of a quadraspheric lens $(\times 1.97$ magnification of laser spot) or an area centralis lens ( $\times 0.94$ magnification of laser spot) (Volk Optical Inc., Mentor, $\mathrm{OH}, \mathrm{USA}$ ). Laser beam diameter was set as 1.2, 2.0 , or $3 \mathrm{~mm}$ according to lesion size, and the power setting was set around 160,260 , or $400 \mathrm{~mW}$, respectively, with area centralis lens. With a quadraspheric lens, the power was increased in proportion to the linear magnification effect of the lens. Laser energy was tested at the retinal periphery to determine the threshold energy level. Subthreshold energy (5-10\% lower than the threshold level) was applied to the CNV membranes. Care was taken to ensure that the entire lesion border plus $500 \mu \mathrm{m}$ was covered with the treatment beam. In patients with large lesions, confluent overlapping treatment was given. Treatment was performed with one spot for $60 \mathrm{~s}$. The end point of treatment was no visible change. If there was extensive fluid elevation or subretinal haemorrhage, the power was increased by $5-10 \%$. Treatment was immediately stopped if any retinal whitening was noted, or if the patient experienced any pain or a burning sensation. In such a case, the remaining course of the 60-s treatment time was completed using half of the initial dosage. Re-treatment was performed if there was no reduction in fluid elevation on slit-lamp biomicroscopy, or if there was persistent leakage noted on angiography 2.5-3 months after the initial treatment. Recurrence was defined as increased exudates or leakage of the previously closed membrane observed by fluorescein angiography.

Best-corrected visual acuity was obtained with Snellen chart measurement and converted to the logarithm of the minimal angle of resolution (logMAR) for statistical analysis. Improvement or decline in vision was defined as change of more than 0.3 in logMAR visual acuity. The cumulative recurrence rates over the follow-up period were calculated with Kaplan-Meier method. Statistical significance was defined as $P<0.05$.

\section{Results}

Of 58 eyes in 55 patients enrolled in this study, there were 37 male $(67.3 \%)$ and 18 female $(32.7 \%)$ patients. The average age was 74.1 years (range: $55-89$ years). In the study patients, 44 membranes were occult $(75.9 \%)$, six classic $(10.3 \%)$, and eight mixed $(13.8 \%)$. The mean follow-up period was $16.6 \pm 10.7$ months (range: $6-48$ months). Initial visual acuity was below $20 / 200$ in 34 eyes (58.6\%), between $20 / 200$ and $20 / 100$ in 14 eyes $(24.1 \%)$, and between $20 / 100$ and $20 / 50$ in 10 eyes (17.3\%).

After TTT, the membranes closed in 46 eyes (79.3\%), according to angiographic examination. Complications 
included subretinal haemorrhage in three eyes (5.2\%), RPE tear in two eyes (3.4\%), and macular area cystic change in two eyes (3.4\%).

In eyes with occult $\mathrm{CNV}$, visual acuity improved in six eyes $(13.6 \%)$, remained unchanged in 14 eyes $(31.8 \%)$, and deteriorated in 24 eyes (54.6\%). For classic type CNV, visual acuity improved in two eyes (33.3\%), remained unchanged in one eye $(16.7 \%)$, and deteriorated in three eyes $(50 \%)$. In eyes with mixed $\mathrm{CNV}$, visual acuity improved in one eye (12.5\%) and deteriorated in the remaining seven eyes $(87.5 \%)$. Overall, $41.4 \%$ eyes had improved or stabilized visual acuity at the end of follow-up. With regard to the different types of $\mathrm{CNV}$, the average logMAR change at the end of follow-up from baseline was 0.30 (SD 0.44) in occult type, -0.08 (SD 0.92) in classic type, and 0.59 (SD 0.77) in mixed type (two-sample Student's $t$-test, $P<0.01)$.

Thirty eyes in this study experienced recurrence $(51.7 \%)$. The interval between treatment and recurrence was 9.2 \pm 6.2 months (range: $2-22$ months). Each patient had an average of 1.97 treatment sessions (range: 1-6 months). Thirty-four eyes $(58.6 \%)$ received more than one treatment session. Using the Kaplan-Meier method, the cumulative recurrence rates were $45 \%$ at 12 months and $71 \%$ at 22 months. Upon comparison of the cumulative recurrence rates between occult type and non-occult type $\mathrm{CNV}$, there was no significant difference (log-rank test, $P=0.715$ ).

\section{Discussion}

The results from our study showed that of 58 eyes with CNV from AMD, at a mean follow-up period of 16.6 months, the best-corrected visual acuity was improved or remained unchanged in $41.4 \%$ eyes. In eyes with occult $\mathrm{CNV}$, the best-corrected visual acuity was improved or remained unchanged in $45.4 \%$. Compared with results from other studies in which visual acuity in $71-82 \%$ eyes was stable or improved, $8,11,12$ the result in our study was less favourable. This difference may be partly attributed to the different baseline visual acuities and the longer average follow-up period in our series. ${ }^{8,11,12}$ In AMD, a gradual visual deterioration over time is expected. Although over half of our patients had decreased vision, the CNV closed in about $80 \%$, and none had increased exudates. Compared with the result of TTT4CNV (E Reichel, TTT4CNV Clinical Trial, ARVO 2005, FL), the largest clinical trial in America, our study showed a greater percentage of cases obtaining visual stabilization. In the TTT4CNV study, the power settings were the same for every patient. Our experience suggested that subthreshold energy level varied greatly among individual patients. Treating patients indiscriminately with a uniform power setting may lead to undertreatment in some patients and overtreatment in others. The natural histories of visual prognosis in occult and classic types CNV are similar. ${ }^{13,14}$ However, our study showed that the visual outcome was better in classic CNV compared to other types. Classic CNV has a well-defined boundary to allow precise targeting. Conversely, occult or mixed type CNV may be associated with serous or haemorrhagic retinal pigment epithelial detachment, (RPED) making energy adjustment difficult and retinal damage possible. Moreover, many occult lesions are large and require more than one spot to cover the entire lesion. Insufficient energy delivery to the spot junction or excessive energy delivery to the area overlapping the spot may occur. These reasons might explain the statistically better visual outcome in classic CNV in this series, although further prospective studies with larger number of cases might be needed.

Adjustment of energy is an important issue in TTT. Fundus pigmentation affects retinal irradiance and subsequent retinal temperature rise. The power settings in our study were approximately half of those used in Caucasian eyes. ${ }^{8,9,10}$ Although we adjusted the energy meticulously to the lower level, there were still complications, including subretinal haemorrhage, RPE tear, and cystic change at the macular area (Figure 1a and $b$ ). All of these complications were associated with a higher level energy. Our complication rate was higher compared to other series. ${ }^{8,10,11}$ An even higher complication rate $(16 \%)$ was noted in a series of Japanese patients using an energy level half of that used in Caucasians. ${ }^{12}$ We may conclude that for light-browncoloured fundus, energy should be adjusted to a lower level to avoid complications. A higher complication rate partly accounts for the less favourable visual outcome in our study.

An RPE tear is a serious complication after TTT. Similar to PDT, the complication most often occurs in treating CNV with serous or haemorrhagic RPED. As in PDT, serous or haemorrhagic RPE should be a contraindication of TTT.

Cystic change at the macular area is a rarely reported complication to our knowledge. One of our patients experienced decreased visual acuity after the fifth application of TTT, and OCT revealed cystic change at the macular area (Figure 2). Two eyes (3.4\%) developed cystic change at the macular area, which was discovered upon follow-up. As no active leakage was noted by fluorescein angiography study in such cases, the cystic changes were more likely associated with repeated TTT treatment. Although TTT has been claimed to be safe to the overlying retina, multiple treatments could be harmful. Macular burn as complication had been 

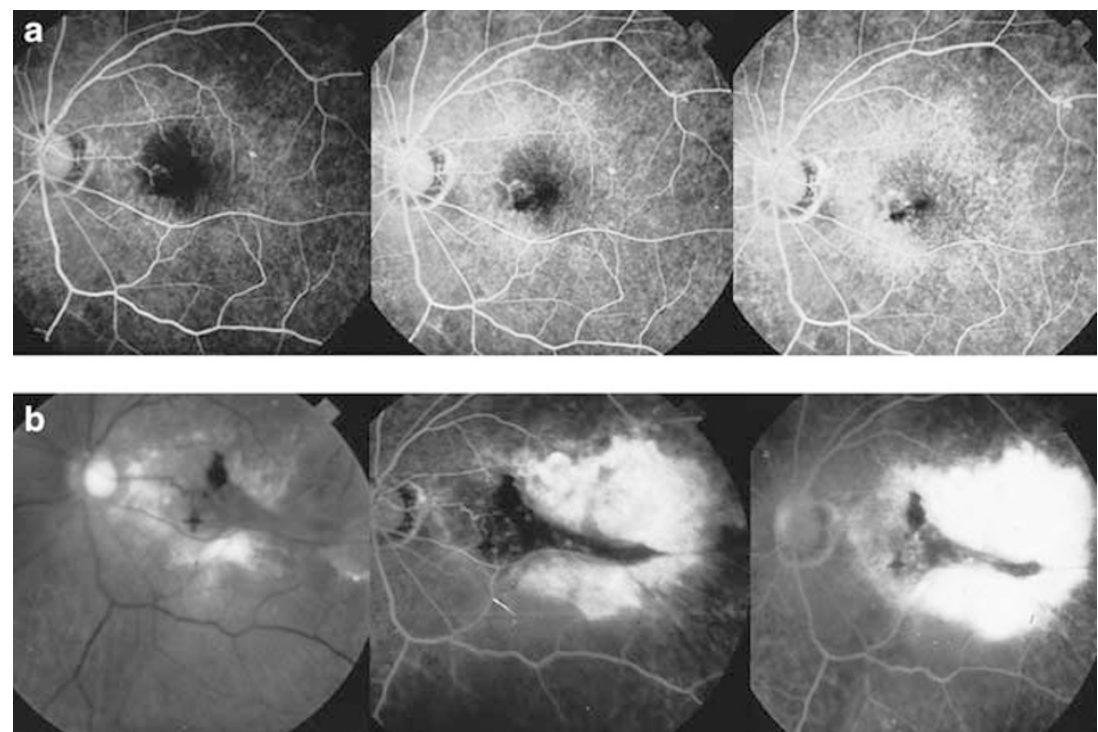

Figure 1 Fluorescein angiography (FA) of an 89-year-old male with occult CNV secondary to AMD. (a) Pre-TTT FA from early to late phase (left to right); neovascular membrane with leakage at late phase was noted. The visual acuity of the left eye was $20 / 50$ before treatment. (b) After two TTT treatments, the visual acuity of the left eye decreased to 20/1000. FA showed RPE tear and extensive leakage at late phase.

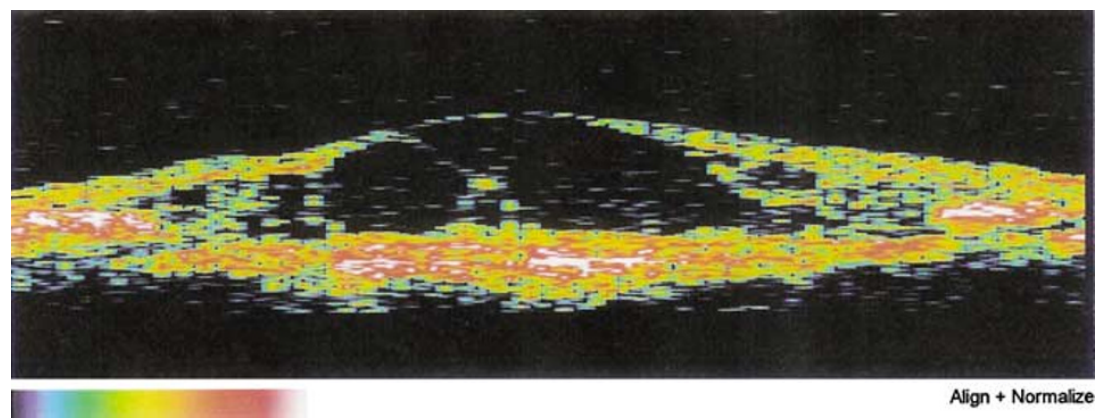

Figure 2 OCT from a 66-year-old male patient with occult CNV secondary to AMD after five TTT treatments. Cystic formation was found at the macular area. Visual acuity declined from $20 / 400$ to finger counting at $40 \mathrm{~cm}$.

reported at a rate of $8.6 \%$ in another series. ${ }^{15}$ Our experience suggests that either the treatment interval should be expanded or the number of treatment session should not exceed four times.

The CNV membranes were closed in 46 (79.3\%) eyes. The closure rate was comparable to other studies (75-94\%). ${ }^{8,10}$ This result suggests that TTT can effectively close CNV in AMD. However, our results showed that the cumulative recurrence rate was high, independent of type of CNV (Figure 3a-c). This is probably the major disadvantage of the treatment. Recently, intravitreal steroid injection has been used as an adjunct to enhance the effect of PDT. Whether the use of steroid via intravitreally or subtenon route may be helpful in TTT treatment to reduce the recurrence rate remains to be seen.
In conclusion, our study concurs that a reduction in late leakage of fluorescein dye, resolution of subretinal blood, and reduction of retinal fluid can be achieved in the majority of the eyes with TTT treatment. However, there is no significant effect of visual improvement or stabilization found. Classic type CNV responds better to TTT. In the light-brown retina, an excess in power setting can be a pitfall, and meticulous adjustment of laser energy according to fundus pigmentation is of great importance. Patients should be informed of the possibility of multiple sessions as well as the possible complications before the treatment. Further prospective study with larger patient numbers and more careful design of power settings should be performed to better assess this treatment modality. 

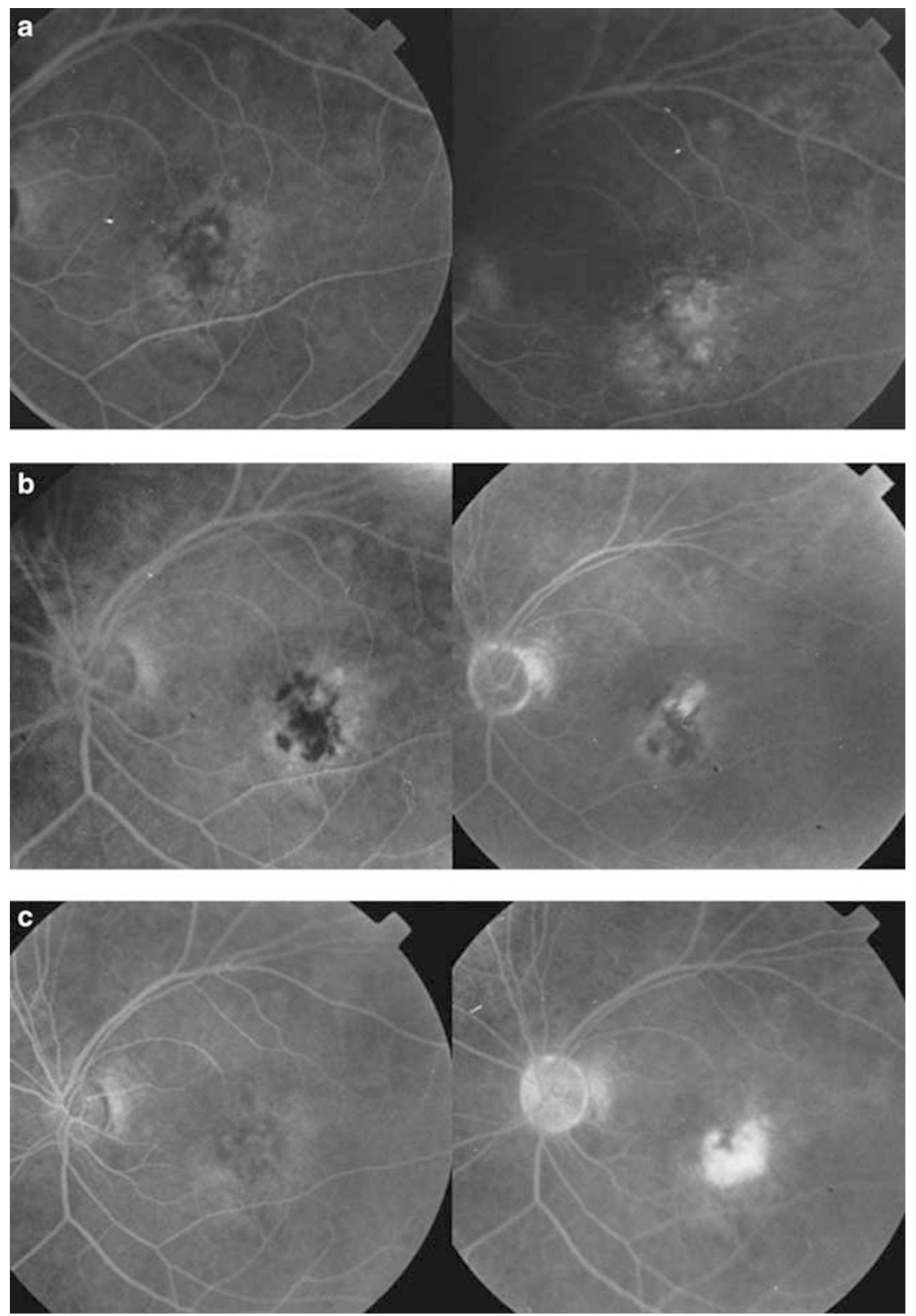

Figure 3 FA of a 74-year-old male with occult CNV secondary to AMD. (a) Pre-TTT FA of early and late phase (left, right). (b) After TTT, late leakage decreased. (c) After 6 months, later, recurrence of CNV leakage was noted.

\section{References}

1 Klein R, Klein BE, Linton KL. Prevalence of age-related maculopathy: the Beaver Dam Eye Study. Ophthalmology 1992; 99: 933-943.

2 Leibowitz HM, Krueger DE, Maunder LR, Milton RC, Kini MM, Kahn HA et al. The Framingham Eye Study monograph: an ophthalmological and epidemiological study of cataract, glaucoma, diabetic retinopathy, macular degeneration, and visual acuity in a general population of 2631 adults, 1973-1975. Surv Ophthalmol 1980; 24: $335-610$
3 Macular Photocoagulation Study Group. Laser photocoagulation of subfoveal neovascular lesions of age-related macular degeneration: updated findings from two clinical trials. Arch Ophthalmol 1993; 111: 1200-1209.

4 Macular Photocoagulation Study Group. Visual outcome after laser photocoagulation for subfoveal choroidal neovascularization secondary to age-related macular degeneration. The influence of initial lesion size and initial visual acuity. Arch Ophthalmol 1994; 112: 480-488.

5 Freund KB, Yannuzi LA, Sorenson JA. Age-related macular degeneration and choroidal neovascularization. Am J Ophthalmol 1993; 115: 786-791. 
6 Bressler NM, Arnold J, Benchaboune M, Blumenkranz MS, Fish GE, Gragoudas ES et al. Verteporfin therapy of subfoveal choroidal neovascularization in patients with age-related macular degeneration: additional information regarding baseline lesion composition's impact on vision outcomes-TAP report No. 3. Arch Ophthalmol 2002; 120: 1443-1454.

7 Bressler NM, Treatment of Age-Related Macular Degeneration with Photodynamic Therapy (TAP) Study Group. Photodynamic therapy of subfoveal choroidal neovascularization in age-related macular degeneration with vertiporfin: two year results of 2 randomized clinical trials - TAP report 2. Arch Ophthalmol 2001; 119: 198-207.

8 Reichel E, Berrocal AM, Ip M, Kroll AJ, Desai V, Duker JS et al. Transpupillary thermotherapy of occult subfoveal choroidal neovascularization in patients with age-related macular degeneration. Ophthalmology 1999; 106: 1908-1914.

9 Mainster MA, Reichel E. Transpupillary thermotherapy for age-related macular degeneration: long-pulse photocoagulation, apoptosis, and heat shock proteins. Ophthalmic Surg Lasers 2000; 31: 359-373.

10 Newsom RS, McAlister JC, Saeed M, McHugh JD. Transpupillary thermotherapy (TTT) for the treatment of choroidal neovascularization. Br J Ophthalmol 2001; 85: 173-178.
11 Thach AB, Sipperley JO, Dugel PU, Sneed SR, Park DW, Cornelius J et al. Large-spot size transpupillary thermotherapy for the treatment of occult choroidal neovascularization associated with age-related macular degeneration. Arch Ophthalmol 2003; 121: 817-820.

12 Atarashi T, Tamaki Y, Inoue Y, Obata R, Muranaka K, Yanagi $\mathrm{Y}$ et al. Transpupillary thermotherapy for treatment of exudative age-related macular degeneration in Japanese patients. Eye 2004; 18: 615-618.

13 Treatment of Age-related Macular Degeneration with Photodynamic Therapy (TAP) Study Group. Photodynamic therapy of subfoveal choroidal neovascularization in agerelated macular degeneration with verteporfin: one-year results of 2 randomized clinical trials - TAP report. Arch Ophthalmol 1999; 117: 1329-1345.

14 Verteporfin in Photodynamic Therapy Study Group. Verteporfin therapy of subfoveal choroidal neovascularization in age-related macular degeneration: two-year results of a randomized clinical trial including lesions with occult with no classic choroidal neovascularization-verteporfin in photodynamic therapy report 2. Am J Ophthalmol 2001; 131: 541-560.

15 Rougier MB, Francois L, Fourmaux E, Isber R, Colin J, Korobelnik JF. Complications and lack of benefit after transpupillary thermotherapy for occult choroidal neovascularization: 1-year results. Retina 2005; 25: 784-788. 